Vol. 18, \#11, Junio 2010

http: // revista-redes.rediris.es

\title{
Desarrollo local y Análisis de Redes Sociales: el valor de las relaciones como factor del desarrollo socioeconómico
}

\author{
Rafael Merinero Rodríguez-Universidad Pablo de Olavide de Sevilla-Centro de \\ Sociología y Políticas Locales ${ }^{1}$
}

\begin{abstract}
Resumen
El modelo de desarrollo socioeconómico ha experimentado en los últimos años una importante transformación, que ha dado lugar al denominado desarrollo local, que viene caracterizado por la importancia que cobran los factores endógenos, propios del territorio, como los elementos básicos en torno a los que articular las acciones de adaptación del mismo a las exigencia del contexto. Este nuevo modelo de desarrollo basado en lo local se asienta en la importancia que cobran los actores y las interacciones de estos para articular propuestas colectivas de adaptación, como lo ha puesto de manifiesto importantes aportaciones teórico-metodológicas, entre las que destaca el Sistema Productivo Local. Desde no hace mucho tiempo, el turismo se ha venido defendiendo como uno de los instrumentos apropiados para articular procesos de desarrollo local, en el que los actores y sus relaciones se convierten en elementos esenciales. Se hace necesario la configuración de modelos analíticos que realmente permitan poner de manifiesto que existe una asociación entre las redes de actores y el nivel de desarrollo, que es precisamente lo que aporta este trabajo. Es decir, la correlación que existe entre las características estructurales de las redes de actores y el nivel de desarrollo turístico de los territorios.
\end{abstract}

Palabras clave: Desarrollo local; desarrollo turístico; redes de actores; Análisis de Redes Sociales.

\begin{abstract}
During the last years, the model of socioeconomic development has undergone an important transformation, that it has generated the so-called local development. This kind of development is characterized by the importance that the endogenous factors have. We speak of these factors, like basic and essential elements surroundings to which we can articulate actions of adaptations to the demands of the new context. This new model of development based in the local elements like essential factors and in the importance that have the social partners and its interactions to articulate collective proposal of adaptation, have been shown in many theoretic and methodological investigations between that it emphasizes the local productive system. Not long time ago, the tourism has been one of the more suitable instrument to articulate processes of local development, where the social partnes and its interaccions are essential elements. We must configurate analitical models that really allows us to show that exist and association between actor nets and the development level in one site. It's just that this essay provides, the correlation between the structural features of actor nets and the level of tourist development in the territories.
\end{abstract}

Key words: Local development, Tourist development, Actor nets, Social network analyses.

${ }^{1}$ Enviar correspondencia a: Prof. Dr. Rafael Merinero Rodríguez rmerrod@upo.es 


\section{I ntroducción}

Ya hace cierto tiempo que desde diferentes ámbitos, algunos de ellos vinculados a la investigación científica y a organizaciones internacionales, se está reclamando que el turismo está desarrollando un cambio amplio de modelo que tiene que ver, principalmente, con el hecho de que se está empezando a considerar como un motor para el desarrollo ${ }^{2}$ local, y que por tanto, implica que el centro de interés se ponga más en la dimensión territorial del mismo, y como extensión, se exponga que los actores locales, los que operan en el territorio, dispongan de un protagonismo especial para impulsar el desarrollo a través del turismo. Esta situación hace que los actores turísticos locales y sus relaciones se conviertan en piezas claves en el nuevo modelo turístico.

Por ello, desde la Organización Mundial del Turismo se ha venido defendiendo que la colaboración entre los actores locales públicos y privados es un elemento clave para articular el desarrollo turístico de un determinado territorio (OMT-WTO, 2001; OMT-WTO, 2002), o lo que en ámbitos académicos se ha venido denominando la gestión activa de los destinos turísticos (Pulido, 2005). El trabajo aquí presentado permitirá comprobar empíricamente cómo, en efecto, existe una evidente correlación entre el nivel de desarrollo turístico de un territorio y las características de las relaciones entre los actores turísticos. De esta forma, se podrá apreciar que a medida que el nivel de desarrollo turístico es más elevado también lo es la densidad y el número de actores centrales de las redes de relaciones de actores con incidencia en la actividad turística en el ámbito local.

El trabajo, por tanto, es pionero por ofrecer un primer modelo de análisis del Sistema Turístico Local (definido este como los actores y sus relaciones) a través de la operacionalización metodológica de las relaciones entre los actores turísticos desde una perspectiva dinámica (un avance científico con respecto a anteriores modelos que se centraban exclusivamente en los factores estáticos de las relaciones), y ha sido aplicado a tres estudios de caso que son ciudades patrimoniales del interior de Andalucía. Es decir, ciudades con unas características

\footnotetext{
2 El concepto de desarrollo utilizado en este trabajo es aquel que lo entiende como crecimiento de la actividad economicoçproductiva de un determinado territorio en terminos de creacion de empleo y rentas, que es el que utiliza la Teoria de los Sistemas Productivos Locales, entendiendolo como los procesos de adaptacion de un determinado territorio a su contexto productivo. Por tanto, se aleja de un concepto de desarrollo mas amplio que lleve a identificarlo con mejora de la calidad de vida en sentido extenso. Asi, en concreto en el ambito del turismo que es el que aquí se analiza el desarrollo turistico se ientifica con el peso que la activiad turistiva tiene en la estrutura de la economia local de cada una de las ciudades analizadas. Por supuesto, hay que ser consciente de que el termino desarrollo es complejo y
} 
similares y que presentan niveles de desarrollo turístico diferentes con el único objetivo de probar las posibilidades analíticas del modelo propuesto. Sin lugar a dudas, el modelo analítico, basado fundamentalmente en el Análisis de Redes Sociales, puede ser aplicado a otros territorios considerados como destinos turísticos más o menos consolidados o a diferentes tipologías de destinos (sol y playa, grandes ciudades, segmentos turísticos), tal es así, que actualmente se está llevando a cabo una investigación en otros tipos de destinos turísticos a nivel nacional.

\section{La Teoría de los Sistemas Productivos Locales: los actores y sus relaciones como aspectos claves del desarrollo local}

El concepto de Sistema Productivo Local está directamente relacionado con el nuevo modelo de desarrollo económico que empieza a prosperar como consecuencia de la crisis del modelo de producción en serie como forma de producción y organización empresarial basado en la gran empresa, adquiriendo en el nuevo modelo un mayor protagonismo las características locales de un determinado territorio: los valores territoriales, de identidad, diversidad y flexibilidad, etc. (Alburquerque, 2004). Esto ha provocado que las estrategias de desarrollo económico del territorio estén evolucionando desde el desarrollo polarizado y el establecimiento de incentivos a la inversión externa, hacia otras estrategias basadas en el aprovechamiento de los recursos endógenos.

Se produce, de esta forma, una respuesta a la insatisfacción que ha generado el agotamiento del modelo de desarrollo "desde fuera" establecido en los años sesenta y setenta basado en la concepción convencional del desarrollo en etapas de Rostow, donde la clave estaba en la imitación mecánica de las sociedades industriales. Frente a este agotamiento aparece el modelo de desarrollo endógeno fundamentado en la evidencia de casos concretos de procesos de desarrollo industrial en territorios determinados y en los intentos de las autoridades públicas de impulsar procesos de desarrollo de localidades sobre la base de las particularidades de los diversos territorios, sus culturas y de sus diferentes sistemas de valores.

El modelo de desarrollo endógeno parte de que todas las comunidades territoriales tienen un conjunto de recursos que constituyen su potencial de desarrollo, lo que hace que en un determinado momento histórico esa colectividad territorial por 
iniciativa propia puede encontrar nuevas ideas y proyectos que le permitan utilizar sus recursos. El concepto de desarrollo endógeno, desde la perspectiva del Sistema Productivo Local, hace referencia a procesos de acumulación de capital en localidades y territorios concretos: se trata de procesos de desarrollo difuso que permite obtener economías de escala externas a las empresas pero internas al sistema productivo, y se producen gracias a la utilización del potencial económico local que favorecen las instituciones y mecanismos de regulación que caracterizan a cada territorio. La forma de organización de la producción, las estructuras familiares, las tradiciones locales, la estructura social y cultural y los códigos de la población condicionan los procesos de desarrollo local. Se concede, por tanto, un papel predominante al territorio, no como soporte físico sino como actor del desarrollo, ya que las empresas, las organizaciones, las instituciones locales y la sociedad civil de un determinado territorio juegan un papel activo en los procesos de crecimiento y cambio estructural (Vázquez, 2000: 95-96). El Sistema Productivo Local es una de las formas en que se concreta el modelo de desarrollo endógeno y tiene que ver con la existencia de concentraciones importantes de pequeñas empresas de una misma actividad industrial en un determinado territorio, siendo lo más característico de todo ello el sistema de relaciones que se establece entre dichas pequeñas y medianas empresas, y la imbricaciones que dichas relaciones tienen con las características sociales y culturales del territorio.

Como corriente teórica, el Sistema Productivo Local tuvo un precedente en la obra de Alfred Marshall, quien al estudiar la organización geográfica de la industria realizó la propuesta de establecer como unidad de estudio del desarrollo económico una entidad de base territorial, denominada distrito. Lo que puso de evidencia Marshall es que el desarrollo industrial no puede reducirse únicamente a la capacidad empresarial, sino que la organización industrial existente en un territorio adquiere un valor significativo.

La línea de trabajo iniciada por Marshall en el sentido de que existe un modo de producción con rendimientos crecientes alternativo al de las grandes empresas integradas de una forma vertical fue continuado por el investigador italiano Becattini, quien puso de manifiesto que existe una alternativa al crecimiento basado en la ubicación de polos de grandes empresas conocidos como "distritos industriales" que está basada en las economías externas generadas por la concentración territorial de pequeñas y medianas empresas especializadas en las

el planteamiento metodologico del trabajo de investigacion. 
diferentes fases de un único proceso productivo (Becattini, 1989). Las empresas, en presencia de economías externas, alcanzan un nivel óptimo de eficiencia si recurren al mercado para obtener aquellos suministros y servicios que en caso de fabricación interna afectarían de forma negativa a los beneficios, configurándose así sistemas industriales locales en torno a una actividad industrial. En la medida en que las economías externas son internas a la industria generan un proceso de localización entre empresas con actividades distintas y complementarias, que en su conjunto forman un mismo proceso productivo y dan lugar a una especialización industrial territorial donde existe un modelo organizativo de la industria basado, simultáneamente, en relaciones de cooperación y competencia. El distrito industrial de Becattini va más allá de la proximidad geográfica y la especialización sectorial destacadas por Marshall, ya que para el funcionamiento óptimo del mismo se destacan los aspectos sociales y culturales del territorio que se convierten en fundamento de las relaciones de colaboración y cooperación entre las empresas (Becattini, 1991). El distrito industrial tiene que ver tanto con una matriz local de interrelaciones técnicas entre empresas como con una red de vínculos socioculturales.

Los distritos industriales de Becattini tuvieron a su vez continuidad teórica en los "medios innovadores" de Aydalot, que pone de manifiesto que la innovación como principio básico de la adaptación de la producción de un territorio no depende exclusivamente de la capacidad de innovación del empresariado sino que es el territorio formado por las empresas, los actores sociales y las administraciones públicas, el que actúa como medio que propicia el emprendimiento y la innovación (Aydalot, 1986). De esta forma se pone en evidencia que en los Sistemas Productivos Locales caracterizados por la existencia de un conjunto importante de pequeñas y medianas empresas éstas no puedan desencadenar un proceso innovador por sí mismas y necesiten acudir a los recursos existentes en el territorio donde se asientan (Caravaca et al, 2002: 41). El esfuerzo para propiciar la generación e incorporación de conocimientos para dar respuesta a los retos y problemas a los que las sociedades deben hacer frente, resulta un factor clave que permite, no sólo a las empresas sino también a los distintos territorios, insertarse con una mejor posición en el contexto caracterizado por la complejidad (Maillat, 1995). Desde esta perspectiva, se considera que la innovación no se puede reducir exclusivamente a la incorporación de innovaciones empresariales de carácter tecnológico sino que debe ser entendida en un sentido amplio como la predisposición a incorporar conocimiento que permita utilizar racionalmente los recursos existentes en cada territorio, y que centrados en los Sistemas Productivos 
Locales deben generar las condiciones para que determinadas necesidades y problemas a los que no pueden responder por sí solas las empresas centradas en el proceso productivo, debe ser proporcionadas por el entorno, por el medio, creando una entorno socio-institucional de actores diferentes a las pequeñas y medianas empresas del distrito que favorezca los procesos de desarrollo (Caravaca y González, 2003).

En cierto sentido, la teoría del Sistema Productivo Local en sus dos variantes, distrito industrial y medio innovador, lo que pone de manifiesto es un nuevo modelo de desarrollo económico, donde el territorio y lo local adquieren un papel preponderante. Los estudios sobre el Sistema Productivo Local han evidenciado que en un determinado territorio se produce una especialización productiva en un tipo de industria particular que permite vislumbrar una trama productiva como conjunto de eslabonamientos entre las diferentes empresas que se encuentran en ese espacio físico a lo largo de las diferentes fases de producción que requiere la tipología productiva en la que se ha especializado el territorio. Desde esta perspectiva, la clave estratégica en cuanto a la competitividad productiva de las empresas se sitúa de manera clara en el nivel de articulación de la red de empresas en torno a un agrupamiento organizado territorial y sectorialmente.

Analizados de esta forma, se constata que los procesos productivos son consecuencia de la integración de múltiples acciones de naturaleza dispar donde intervienen un número importante de agentes empresariales que requieren de una proximidad física relacional, implicando la configuración de unidades productivas territoriales. En el centro del proceso de crecimiento y acumulación de capital de los sistemas productivos locales está la forma de organización del sistema productivo que propicia la formación de economías de escala externas y la reducción de los costes de transacción, lo que hace que la dinámica de la economía local gire alrededor de la organización del sistema productivo, en el que se establecen las relaciones entre las empresas, los proveedores y los clientes. La columna vertebral de los sistemas productivos locales es la configuración del modelo de producción, la existencia de una red de empresas industriales que dan lugar a una multiplicidad de mercados internos y de intercambios a escala local; las relaciones dentro de la red no sólo permiten el intercambio de productos y servicios entre los actores sino también de conocimientos tecnológicos y de pautas de comportamiento, de manera que las relaciones se basan en el conocimiento que unos actores tienen de otros, en la confianza mutua que se ha ido generando paulatinamente, así como en el beneficio que el comercio e intercambio produce (Vázquez, 2000: 98). 
Esto, sin duda, hace que el modelo de desarrollo que le es propio al Sistema Productivo Local se caracterice por su dimensión territorial local, no sólo debido al efecto espacial de los procesos organizativos y tecnológicos, sino por el hecho de que cada localidad, cada región, es el resultado de una historia en la que se ha ido configurando el entorno institucional, económico y organizativo; cada espacio económico aparece con una configuración propia, que se ha ido definiendo en función de los sucesivos sistemas productivos, de los cambios organizativos de las empresas e instituciones y de las transformaciones en el sistema de relaciones sociales e industriales (Vázquez, 1999). Esto ha provocado que a la teoría de los Sistemas Productivos Locales se le haya denominado como Teoría Territorial de la Cooperación, en la que las redes se ven como una alternativa al mercado, menos costosa en términos de identificación, acceso e intercambio de bienes, servicios o conocimientos entre empresas debido a que la pertenencia a un mismo ámbito espacial, en el que existe una cierta homogeneidad idiomática, cultural e institucional y en el que los intercambios se convierten en algo habitual, genera un clima de confianza y entendimiento que ayuda a reducir los comportamientos indebidos.

En la literatura científica reciente sobre el turismo se está poniendo de manifiesto la importancia que tiene atender a la denominada gestión activa del destino como elemento fundamental de la competitividad del turismo. Poner el foco de atención en la gestión implica en primer lugar realizar un esfuerzo en operacionalizar analíticamente dicho concepto, y establecer, por tanto cuales serán las dimensiones a las que habrá que prestarle interés para su comprensión en profundidad.

Desde no hace mucho tiempo han venido apareciendo un conjunto de trabajos científicos sobre el turismo que tienen que ver con la aplicación de la Teoría de los Sistemas Productivos Locales, que ponen el acento en el territorio y el centro de interés en los actores y las relaciones productivas que se dan entre ellos y que se concreta en los denominados estudios de Clusters Turísticos (Michael, 2007; Lazzaretti y Petrillo, 2006; Novelli, Schmitz y Spencer, 2006; Pforr, 2006; Pavlovch, 2003; Dredge, 2006; Hsin-Yu, 2006; Vernon, Essex, Pinder y Curry, 2005). También es necesario poner de manifiesto que ha aparecido una línea de trabajo que se ha aplicado al estudio de las relaciones entre actores turísticos en este marco de la gestión activa de los destinos, y que tiene que ver con la aplicación de la denominada Teoría de los Stakeholders (Aas, Ladkin y Fletcher, 2005; Bramwell y Lane, 1999; Medeiros y Bramwell, 2002; Sautter y Leiden, 1999; Selin y Chavez, 1995; Yuksel, Bramwell y Yuksel, 1999). 
Sin embargo, estos trabajos a pesar de que han supuesto un avance científico en el análisis del turismo presentan un déficit analítico. El avance tiene que ver con el punto de mira para la comprensión de la actividad turística al centrase en un territorio concreto y conseguir caracterizar al Sistema Turístico a través de sus dos elementos básicos, que son los actores que intervienen en la actividad turística de dicho territorio y las relaciones productivas que se dan entre ellos. Y el déficit tiene que ver, precisamente, con el escaso tratamiento analítico que se le proporciona a las relaciones que efectivamente se producen en el territorio para abordar actividades turísticas. De esta manera, se puede destacar que los análisis propuestos en dichos trabajos no permiten analizar la dinámica real de las relaciones entre los actores, es decir, la dinámica de colaboración que efectivamente se produce entre aquellos actores que realmente interactúan para desarrollar actividades turísticas en el proceso productivo del turismo en un determinado territorio, sea éste un destino consolidado o en formación. Si realmente se quiere abordar el estudio de la gestión activa del turismo en un determinado territorio es necesario que se preste atención a estos factores dinámicos de las relaciones entre los actores que son el fundamento de las características del proceso productivo del turismo. Por la especificidad de dicho proceso productivo de la actividad turística, en el que la colaboración y las relaciones se convierten en un aspecto más que fundamental, tener capacidad de atender a estas dinámicas colaborativas reales que se producen entre los actores públicos y privados que operan en el territorio es el elemento clave para comprender el papel que la gestión activa del turismo tiene en el desarrollo del mismo en el territorio concreto en el que se produce

\section{Metodología de investigación: en busca de las relaciones}

Como ha sido destacado en el apartado anterior, la finalidad principal del trabajo de investigación que aquí se presenta estriba en determinar la importancia que las relaciones entre los actores turísticos tienen en la configuración de las características del destino turístico, en la conformación del denominado producto turístico territorial, es decir, en determinar cómo funciona turísticamente un territorio; o para mayor concreción y utilizando términos analíticos tradicionales del análisis del turismo, se centra en el estudio del funcionamiento de la oferta turística de un determinado territorio, cómo funciona y en que se concreta la oferta de producto turístico de dicho territorio. Tomar este aspecto como punto de partida de una investigación ha tenido como resultado que el planteamiento de la misma se ubique, de una forma decidida, en la convicción científica de que las relaciones 
entre los actores, y no sólo los atributos como manifestación de la estructura social, tiene un poder explicativo importante del comportamiento social (Molina, 2001).

Desde esta perspectiva parece lógico que el planteamiento metodológico de la investigación gire en torno a la importancia que las relaciones entre los actores turísticos de un determinado territorio tienen para explicar las características de la oferta turística del mismo; y que por tanto este planteamiento se centre de una forma prioritaria en buscar las relaciones entre dichos actores turísticos.

Los elementos principales de la nueva línea de trabajo que se está proponiendo aquí, lo componen los actores que intervienen en la actividad turística en un territorio determinado y las relaciones que establecen entre ellos en el curso de las acciones que es necesario llevar a cabo para gestionar turísticamente dicho territorio. Si bien es cierto que la identificación de los actores turísticos y su caracterización no presenta una excesiva complejidad, no ocurre lo mismo con el tratamiento de sus relaciones, y es precisamente en esto, en la operacionalización de las relaciones, donde se encuentra el principal fundamento (y la aportación metodológica) de esta nueva línea de trabajo para el estudio del turismo. Para proceder al tratamiento científico de las relaciones en el Sistema Turístico Local se parte del concepto de proceso productivo turístico del territorio, que tiene por objeto establecer cuáles son las dimensiones básicas de acción a realizar en un determinado territorio para que funcione como un producto o destino turístico. A este respecto el proceso productivo se conforma en cuatro grandes ámbitos de acción (creación, promoción, comercialización y articulación o gestión del producto) en cada uno de los cuales se incluye un número determinado de acciones turísticas concretas que son susceptibles de ser realizadas en colaboración por los distintos actores, es decir que están configuradas por la interacción entre ellos. De esta forma, para el ámbito de la gestión de los destinos turísticos las actividades que son susceptibles de ser realizadas en un proceso de colaboración entre los actores son:

a) En la creación del producto turístico

- Construcción de infraestructuras de acceso a los destinos

- Puesta en valor turístico de recursos culturales y naturales

- Creación de equipamientos y adecuación para la visita

- Creación de servicios turísticos

- Organización de eventos 
- Creación de sistemas normativos

- Creación de sistemas de producción y acceso a materias primas

- Acciones de innovación

b) En la gestión del producto turístico

- Diseño de paquetes turísticos

- Receptivo turístico

- Apertura con personal especializado

- Apoyo a la gestión de la atención turística a través del desvío de clientes

- Señalización

- Servicio de guías turísticos

- Guías turísticas en diferente formato

- Bonos turísticos

- Organización de sistemas de transporte para turistas

- Aplicación de sistemas de calidad

c) En la promoción turística

- Asistencia a ferias

- Asistencia a Work Shops

- Edición de material promocional

- Campañas publicitarias

d) En la comercialización turística

- Comercialización directa

- Comercialización indirecta

Por tanto, los datos necesarios para un análisis de este tipo son los relativos a las relaciones entre los actores que forman parte del sistema y que se obtienen a partir de la detección de las interacciones entre los actores turísticos que operan en un determinado territorio. Para obtener la información relacional necesaria en este modelo analítico se hace imprescindible atender a las características de acción de dichos actores turísticos. De esta forma, se consideran como tales, las administraciones públicas locales, provinciales y regionales; hoteles; restaurantes; 
empresas de servicios turísticos; y asociaciones de la sociedad civil cuya actividad afecta directamente al turismo del territorio, así como el análisis de documentos que informan sobre las actividades turísticas que se desarrollan. La siguiente tabla puede ser ilustrativa de las técnicas de investigación utilizadas.

\begin{tabular}{|c|c|c|c|}
\hline Técnica & I nstrumento & Población & Objetivo \\
\hline $\begin{array}{l}\text { Entrevista informante } \\
\text { clave }\end{array}$ & Guión informativo & $\begin{array}{l}\text { Persona con } \\
\text { conocimiento profundo } \\
\text { del turismo }\end{array}$ & $\begin{array}{l}\text { Identificar a todos los } \\
\text { actores turísticos } \\
\text { Establecer tipología de } \\
\text { actores }\end{array}$ \\
\hline $\begin{array}{l}\text { Entrevista } \\
\text { semiestructurada a } \\
\text { informantes tipo }\end{array}$ & Ámbitos temáticos & $\begin{array}{l}\text { Personas } \\
\text { representativas de los } \\
\text { diferentes tipos de } \\
\text { actores turísticos }\end{array}$ & $\begin{array}{l}\text { Determinar las } \\
\text { características básicas y } \\
\text { los componentes de } \\
\text { naturaleza de los } \\
\text { diferentes tipos de } \\
\text { relaciones turísticas } \\
\text { que se establecen }\end{array}$ \\
\hline $\begin{array}{l}\text { Entrevista con } \\
\text { cuestionario a los } \\
\text { actores turísticos } \\
\text { (Método de redes } \\
\text { completas) }\end{array}$ & Cuestionario & $\begin{array}{l}\text { Todos los actores } \\
\text { turísticos que tienen } \\
\text { incidencia en la } \\
\text { actividad turística del } \\
\text { territorio }\end{array}$ & $\begin{array}{l}\text { I dentificar } \\
\text { características de las } \\
\text { relaciones entre los } \\
\text { actores turísticos del } \\
\text { territorio }\end{array}$ \\
\hline $\begin{array}{l}\text { Datos secundarios } \\
\text { (Método de redes } \\
\text { completas) }\end{array}$ & $\begin{array}{l}\text { Informes, material } \\
\text { informativo, folletos } \\
\text { divulgativos, páginas } \\
\text { Web, etc. }\end{array}$ & $\begin{array}{l}\text { Todo el material } \\
\text { existente de la ciudad } \\
\text { que pueda ser } \\
\text { localizado en las } \\
\text { reuniones y entrevistas } \\
\text { con actores }\end{array}$ & $\begin{array}{l}\text { I dentificar } \\
\text { características de las } \\
\text { relaciones entre los } \\
\text { actores turísticos del } \\
\text { territorio }\end{array}$ \\
\hline
\end{tabular}

Cuadro 1. Técnicas de investigación utilizadas

Fuente: elaboración propia.

Así, mediante la aplicación de dichas técnicas de investigación se puede obtener toda la información relacional, de manera que se puedan conseguir los datos sobre las relaciones que se establecen entre los diferentes actores turísticos de un determinado territorio.

Una vez obtenidos los datos relacionales, se procedió al análisis de los mismos, y para ello se utilizó la técnica del Análisis de Redes Sociales a través del programa de tratamiento de datos relacionales UCINET 6 que permite identificar las características estructurales de las redes de actores (Borgatti, Everett y Freeman, 2002). Sobre la base del Método de Redes Completas en el que se incluye a todo el censo de actores y todas las relaciones posibles entre ellos y las medidas binarias de relaciones (si se producen o no entre los actores), se obtiene el grueso de datos relacionales necesarios para un estudio de redes sociales (Hanneman y Riddle, 2005). A partir de estos datos, el programa de tratamiento de datos relacionales permite elaborar una Matriz de Adyacencia, que es el soporte matemático sobre el 
que se graba la información real de las relaciones entre todos los actores (Quiroga, 2003). Y sobre la información de la Matriz de Adyacencia se pueden obtener una serie de indicadores sobre la estructura formal de la red de relaciones. Para el caso que se presenta aquí se han utilizado tres tipos distintos de indicadores: 1) la densidad de la red que permite medir la proporción de relaciones existentes sobre el total de relaciones posible, indicando, por tanto, la intensidad de las relaciones en el conjunto de toda la red (Rodríguez y Mérida, 2005); 2) el grado de centralidad, que es el número de actores a los que un actor está directamente unido, y la dirección de las relaciones, que pueden ser de salida (las relaciones que un actor tiene con otros actores), y de entrada (el número de actores que tienen relaciones con él). El programa de tratamiento de datos relacionales UCINET 6 aporta la posibilidad de construir el gráfico de la red de actores, en el que se pueden representar las características de las relaciones de dicha red, las posiciones que ocupan los actores y las líneas que marcan y determina las posiciones de cada uno de los actores de la red.

\section{Desarrollo local, turismo y redes de actores en las Ciudades Medias del interior de Andalucía: la importancia de las relaciones en el desarrollo turístico}

Con el objetivo de comprobar la potencialidad del modelo analítico propuesto(las redes de colaboración público-privadas para la realización de actividades productivas en el ámbito del turismo de un determinado territorio) para explicar el desarrollo turístico de los territorios, se ha aplicado la metodología descrita al análisis comparado la actividad turística de tres ciudades medias del interior de Andalucía con marcado carácter patrimonial: Antequera, en la provincia de Málaga; Écija, en la provincia de Sevilla; y Úbeda, en la provincia de Jaén. La tabla siguiente refleja algunos de los aspectos relevantes a los efectos de este estudio de cade una de estas ciudades.

\begin{tabular}{|c|c|c|c|c|c|}
\hline & $\begin{array}{l}\text { Población } \\
(2006)^{a}\end{array}$ & $\begin{array}{c}\text { Recursos }^{\mathrm{d}} \\
\text { patrimoniales }^{\mathrm{d}}\end{array}$ & $\begin{array}{l}\text { Actores } \\
\text { turísticos }\end{array}$ & $\begin{array}{c}\text { Plazas de } \\
\text { alojamiento }^{\mathrm{d}}\end{array}$ & $\begin{array}{l}\text { Visitantes } \\
\text { (2006) }\end{array}$ \\
\hline Écija & 39.295 & 42 & 30 & 258 & $11.188^{\mathrm{b}}$ \\
\hline Antequera & 44.032 & 54 & 55 & 1.910 & $45.307^{b}$ \\
\hline Úbeda & 34.139 & 51 & 49 & 817 & $130.764^{c}$ \\
\hline
\end{tabular}

Fuentes: ${ }^{a}$ padrón municipal de habitantes, instituto nacional de estadística; ${ }^{b}$ oficina de información turística; ${ }^{c}$ turismo andaluz, s.a.; ${ }^{\mathrm{d}}$ elaboración propia 
El objetivo del estudio comparado es identificar si las redes de actores públicoprivadas que definen cada uno de estos Sistemas Turísticos Locales tiene una relación con el nivel de desarrollo turístico que presentan, teniendo en cuenta que son tres casos de similares características en cuanto a su población, a su ubicación geográfica, a su papel histórico en la estructuración territorial de Andalucía, con una riqueza de patrimonio cultural similares, y que, sin embargo, presentan distintos niveles de desarrollo turístico. Para determinar el índice de desarrollo turístico se ha optado por utilizar un indicador para medir la importancia del turismo en relación con la estructura económica de cada una de las tres ciudades analizadas; en concreto se ha utilizado el Índice Turístico elaborado por el Servicio de Estudios de La Caixa que se obtiene en función de la cuota o Impuesto de Actividades Económicas correspondiente a las actividades turísticas, el cual se basa a su vez en la categoría de los establecimientos turísticos (hoteles y moteles, hoteles-apartamentos, hostales y pensiones, fondas y casas de huéspedes, campings y apartamentos gestionados por empresas), número de habitaciones y ocupación anual (todo el año o parte del año); por lo que constituye prácticamente un indicador de la oferta turística (La Caixa, 2007).

\begin{tabular}{|l|c|c|c|}
\hline \multicolumn{4}{|c|}{ Í ndice turístico de las ciudades estudiadas } \\
\hline Ciudad & Écija & Antequera & Úbeda \\
\hline Índice turístico & 10 & 38 & 47 \\
\hline $\begin{array}{l}\text { Fuente: anuario económico de españa. } 2007 \text { (la caixa, } \\
\text { 2007) }\end{array}$ \\
\hline
\end{tabular}

Los estudios tradicionales de competitividad tendrían en cuenta factores del tipo de la localización geográfica, la facilidad de acceso o la riqueza de su patrimonio cultural como aspectos indicativos de su nivel de desarrollo turístico o de la potencialidad turística de la ciudad. En cuanto a la localización es Écija la ciudad mejor ubicada por su cercanía a grandes núcleos de población y su mejor dotación de infraestructuras de comunicación; Antequera ocupa el segundo lugar según este criterio, y úbeda el tercero. La misma clasificación se podría realizar atendiendo a los recursos patrimoniales y el número de Bienes de Interés Cultural. Sin embargo, es la ciudad de úbeda la que presenta un mayor nivel de desarrollo turístico, seguida de Antequera y en tercer lugar de Écija. Se puede afirmar en consecuencia que el desarrollo turístico de estas ciudades no depende de la ventaja de localización o de su riqueza patrimonial, sino que parece depender más de las estrategias seguidas por los actores que participan en la actividad turística de cada 
una de esas ciudades: la naturaleza y las características de las redes que forman en su actuación.

El estudio de las redes de actores turísticos existentes en cada una de las ciudades (que se representan en las páginas siguientes) según el método de Análisis de Redes Sociales, puede aportar alguna solución a este problema.

\section{La red de actores turísticos de la ciudad de Écija}

A partir del gráfico que aparece en la página siguiente se pueden establecer las características básicas de las relaciones entre los actores turísticos de la ciudad de Écija. La primera de las características es que son muy pocas las relaciones que se establecen entre los actores turísticos de la ciudad de Écija. Como recoge el gráfico, todas las relaciones que se producen entre los actores de Écija en todas los ámbitos que componen el proceso de producción turística de la ciudad. Y el aspecto que más destaca es la escasez de relaciones que se produce entre dichos actores, como queda demostrado en la densidad de la red, que es sólo de 18,85\% de todas las relaciones posibles. Este indicador pone de manifiesto claramente que son muy pocas las relaciones que se establecen entre los diferentes actores que intervienen en el Sistema Turístico Local de la ciudad de Écija. Esto demuestra, por una parte que no para todos los actores turísticos de la ciudad es importante el turismo como actividad económica, y que por tanto no intervienen en las relaciones necesarias para crear, gestionar, promocionar o comercializar turísticamente la ciudad, ya que consideran el turismo como una actividad secundaria, y a veces anecdótica de su iniciativa empresarial, como queda demostrado por la nula implicación de la mayoría de los hoteles de la ciudad y de los restaurantes más clásicos; y por otra parte, existe otro importante conjunto de actores que aunque si consideran importante el turismo para su actividad son poco activos como puede ser el caso de los tres museos privados o la empresa de gestión de visitas Veca. 


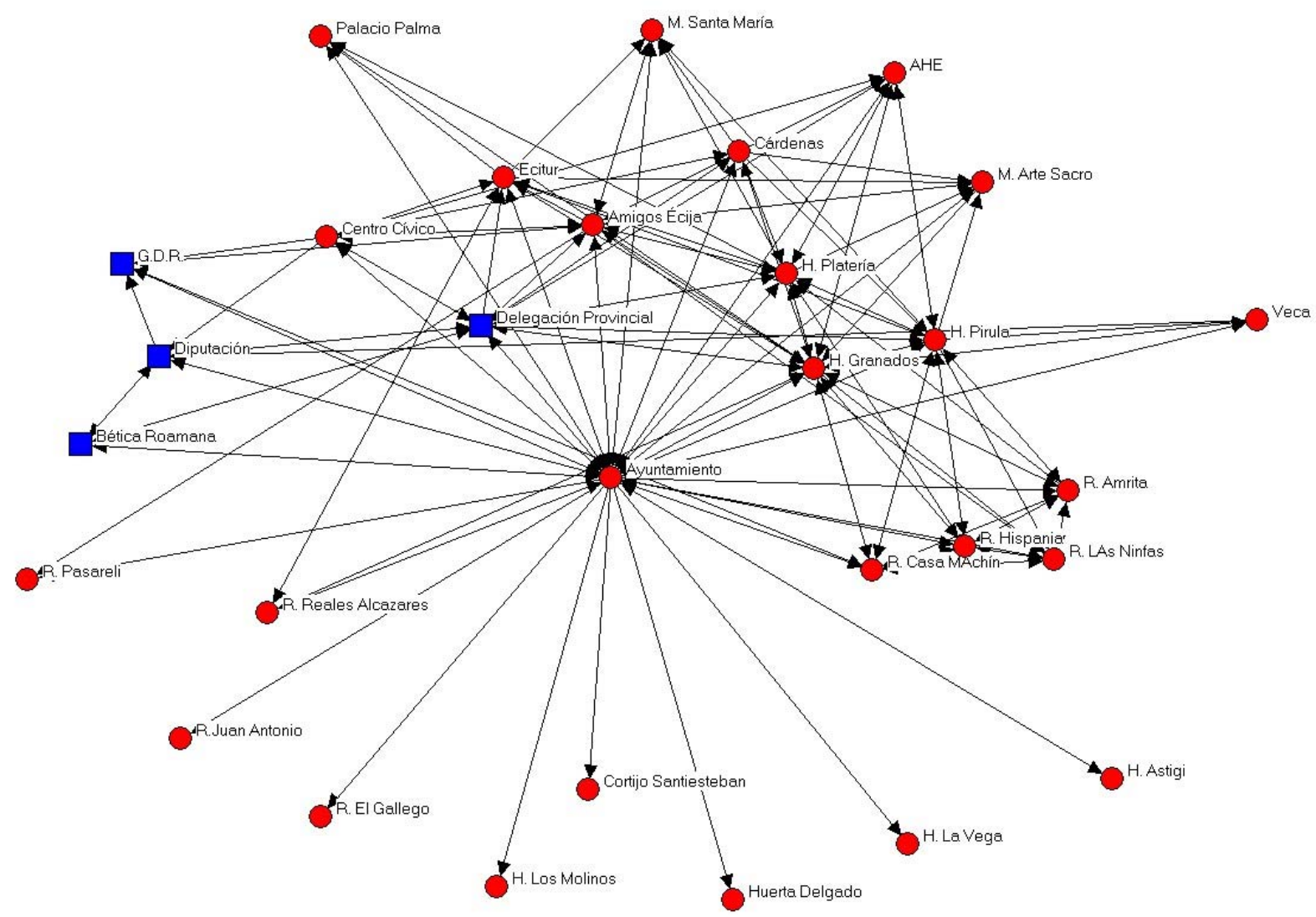

Gráfico 1. Red de actores turísticos de la ciudad de Écija.

La tabla siguiente contiene el grado de centralidad normalizado de entrada y de salida de los actores turísticos de la ciudad de Écija.

\begin{tabular}{|l|c|c|}
\hline \multicolumn{1}{|c|}{ Actor } & $\begin{array}{c}\text { Grado de centralidad de } \\
\text { salida normalizado }\end{array}$ & $\begin{array}{c}\text { Grado de centralidad de } \\
\text { entrada normalizado }\end{array}$ \\
\hline Ayuntamiento & 100 & 75,862 \\
\hline Hotel Granados & 48,276 & 44,828 \\
\hline Hotel Pirula & 44,828 & 31,034 \\
\hline Amigos de Écija & 37,931 & 34,438 \\
\hline Delegación Prov. De Turismo & 37,931 & 31.034 \\
\hline Cárdenas & 27,585 & 13,793 \\
\hline R. Casa Machín & 27,586 & 24,138 \\
\hline Centro Cívico & 27,585 & 10,345 \\
\hline Ecitur & 27,586 & 27,586 \\
\hline H. Platería & 24,138 & 37,931 \\
\hline R. Hispania & 24,138 & 20,690 \\
\hline R. Amrita & 24,138 & 20,690 \\
\hline AHE & 20,690 & 20,690 \\
\hline R. Las Ninfas & 13,793 & 24,138 \\
\hline
\end{tabular}




\begin{tabular}{|l|c|c|}
\hline Diputación Provincial & 13,793 & 17,241 \\
\hline R. Reales Alcázares & 10,345 & 6,897 \\
\hline Museo de Arte Sacro & 10,345 & 20,690 \\
\hline Grupo de Desarrollo Rural & 10,345 & 17,241 \\
\hline Veca & 10,345 & 10,345 \\
\hline Bética Romana & 10,345 & 10,345 \\
\hline Museo de Santa María & 6,897 & 20,690 \\
\hline H. Astigi & 3,448 & 3,448 \\
\hline Palacio de los Palma & 3,448 & 13,793 \\
\hline R. El Gallego & 0 & 3,448 \\
\hline H. Los Molinos & 0 & 3,448 \\
\hline R. Juan Antonio & 0 & 3.448 \\
\hline R. Pasareli & 0 & 6.897 \\
\hline Cortijo Santiesteban & 0 & 3,488 \\
\hline H. La Vega & 0 & 3,488 \\
\hline Huerta Delgado & 0 & 3,488 \\
\hline
\end{tabular}

Tabla 1. Grado de centralidad normalizado de los actores turísticos de écija

Fuente: Elaboración propia

Como se puede comprobar a través de dichos datos, que complementan a las características estructurales que aporta la representación gráfica de dicha red de actores, tan sólo existe un actor con un grado de centralidad de salida y de entrada importante, esta actor es el Ayuntamiento de la ciudad; este aspecto condiciona de manera fundamental la estructura formal que presenta esta red de relaciones, ya que su apariencia es claramente de tipo estrella, con un claro actor central que lidera todas las relaciones y que presenta una clara influencia, ya que a él recurren todos los demás a la hora de impulsar acciones turísticas conjuntas.

Del mismo modo, también se puede apreciar un pequeño grupo de actores, en concreto cuatro actores que son los dos hoteles del centro de la ciudad, la Asociacion Amigos del Museo de Ecija y la Delegacion Provincial de Sevilla de la Consejeria de Turismo, Comercio y Deporte de la Junta de Andalucia, que tienen un grado de centralidad de salida relativamente alto, aunque no llega al 50\%, que se sitúa entre el $50 \%$ y el $30 \%$, y que gráficamente están representados en la parte derecha superior del gráfico de la red. Estos actores impulsan algunas vinculaciones con otros actores para el desarrollo de acciones conjuntas en el ámbito turístico de la ciudad de Écija, pero son relativamente con pocos actores, como se puede comprobar en el indicador del grado de centralidad de entrada, que tampoco presenta datos elevados, situándose en las mismas proporciones (es decir, entre el $50 \%$ y el $30 \%)$. 
Existe otro grupo de actores, el segundo más numeroso, formado por los restaurantes que estan ubicados en el centro historico de la ciudad, la empresa de guias y un centro de actividades ecuestres, que presentan un grado de centralidad de entrada y salida bajo, que se sitúa entre el $20 \%$ y el $30 \%$, y que por lo tanto, están implicados en muy pocas interacciones para el desarrollo de actividades turísticas realizadas de forma conjunta, y que gráficamente están en la segunda línea de actores en la parte derecha y arriba de la red. Y el grupo más numeroso es aquel que tiene un grado de centralidad de salida muy bajo, que apenas si alcanza el $13 \%$, y que en términos muy parecidos se encuentra el grado de centralidad de salida. Además, también se puede comprobar que existe un número relativamente importante de actores que no tienen grado de centralidad de salida, y que por tanto, no impulsan actuaciones turísticas de forma conjunta.

En conclusión, para el caso de la red de actores de Écija, se puede mantener que ésta tiene una estructura clara en forma de estrella, en el que prácticamente un único actor impulsa relaciones con los otros actores para el desarrollo de acciones conjuntas en el marco del turismo en dicha ciudad.

\section{La red de actores turísticos de la ciudad de Antequera}

Respecto a la red de actores turísticos de la ciudad de Antequera, el primero de los aspectos a destacar es la densidad que presenta la red, y hay que decir a este respeto, que se trata de una densidad considerablemente importante, ya que se sitúa en el $29,25 \%$, es decir, que se producen de forma real casi el $30 \%$ de las relaciones posibles entre los actores del Sistema Turístico Local de Antequera. Este dato, junto con la imagen que proporciona el gráfico de la red, pone de manifiesto que son muchos los actores que se encuentran inmersos en interacciones con otros actores para promover y realizar acciones turísticas en Antequera, y no sólo son muchos los actores implicados activamente en la red, sino que entre ellos se produce un volumen importante de relaciones, que es lo que permite explicar la densidad tan importante que presenta la red de actores turísticos de esta ciudad.

En la página que aparece a continuación se encuentra la representación gráfica de la red de actores turísticos de Antequera, que conjuntamente con el grado normalizado de centralidad de salida y entrada puede ayudar a comprender las características estructurales que presenta dicha red. 


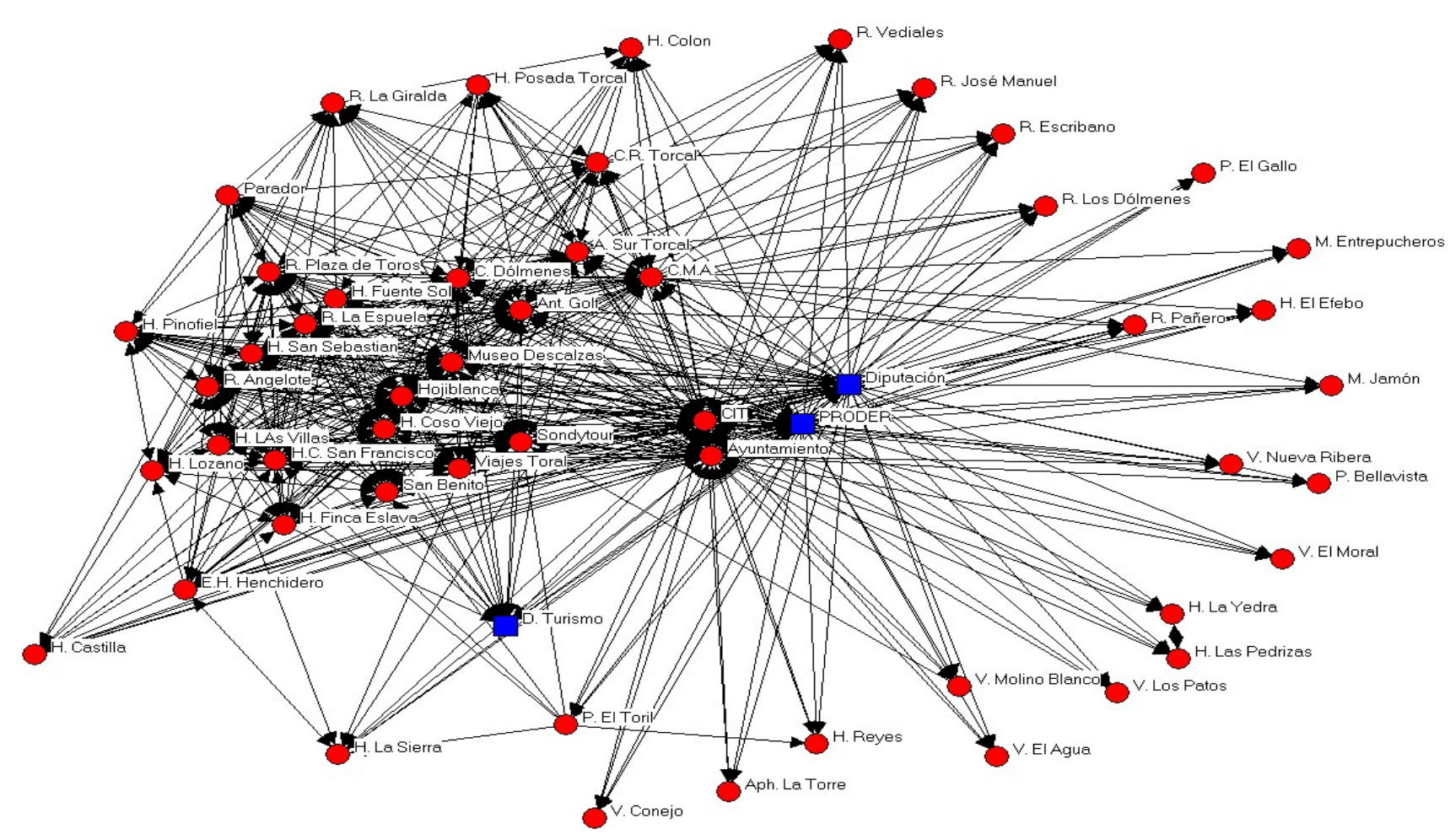

Gráfico 2. Red de actores turísticos de la ciudad de Antequera.

La tabla que aparece a continuación contiene los grados normalizados de centralidad de salida y entrada para los actores turísticos de la ciudad de Antequera, que pueden ayudar a clarificar las características estructurales que presenta la red de relaciones en dicha ciudad.

\begin{tabular}{|l|c|c|}
\hline \multicolumn{1}{|c|}{ Actor } & $\begin{array}{c}\text { Grado de centralidad de } \\
\text { salida normalizado }\end{array}$ & $\begin{array}{c}\text { Grado de centralidad de } \\
\text { entrada normalizado }\end{array}$ \\
\hline Ayuntamiento & 100 & 100 \\
\hline Centro Iniciativas Turísticas & 100 & 100 \\
\hline PRODER & 100 & 41,509 \\
\hline Diputación & 98,113 & 9,434 \\
\hline Consejería de Medio Ambiente & 66,038 & 33,962 \\
\hline Antequera Golf & 62,264 & 54,717 \\
\hline Complejo Arqueológico Dólmenes & 49,057 & 52,830 \\
\hline H. Fuente del Sol & 49,057 & 30,189 \\
\hline Sondytur & 45,283 & 52,830 \\
\hline Asociación Sur Torcal & 45,283 & 47,170 \\
\hline Viajes Toral & 43,396 & 50,943 \\
\hline H. San Sebastián & 43,396 & 43,396 \\
\hline Cooperativa Hojiblanca & 43,396 & 52,830 \\
\hline Museo de las Descalzas & 43,396 & 52,830 \\
\hline H. Finca Eslava & 41,509 & 43,396 \\
\hline
\end{tabular}




\begin{tabular}{|c|c|c|}
\hline H. Lozano & 41,509 & 41,509 \\
\hline H. Coso Viejo & 41,509 & 49,057 \\
\hline R. San Benito & 41,509 & 50,943 \\
\hline Hospedería San Francisco & 41,509 & 45,283 \\
\hline H. Las Villas & 41,509 & 49,057 \\
\hline R. La Espuela & 39,623 & 47,170 \\
\hline R. Angelote & 39,623 & 49,057 \\
\hline R. Plaza de Toros & 39,623 & 50,943 \\
\hline H. Pinofiel & 39,623 & 45,283 \\
\hline Parador Nacional & 35,849 & 15,094 \\
\hline Complejo Rural El Torcal & 33,962 & 18,868 \\
\hline Escuela de Hostelería El Henchidero & 24,528 & 26,415 \\
\hline Hotel Posada Torcal & 22,642 & 15,094 \\
\hline Delegación de Turismo & 16,981 & 28,302 \\
\hline H. Castilla & 15,095 & 16,981 \\
\hline Pensión El Toril & 15,094 & 7,547 \\
\hline H. Colón & 15.094 & 15,094 \\
\hline R. La Giralda & 13,208 & 28,302 \\
\hline H. La Sierra & 5,660 & 16,981 \\
\hline H. La Yedra & 5,660 & 9,434 \\
\hline Venta Molino Blanco & 5,660 & 7,547 \\
\hline R. Los Dólmenes & 5,660 & 13,208 \\
\hline H. Las Pedrizas & 5,660 & 9,434 \\
\hline H. El Efebo & 3,744 & 9,434 \\
\hline Pensión El Gallo & 3,744 & 7,547 \\
\hline R. Pañero & 3,744 & 11,321 \\
\hline H. Reyes & 3,744 & 9,434 \\
\hline Venta Nueva Ribera & 3,744 & 7,547 \\
\hline Venta Los Patos & 3,744 & 7,547 \\
\hline Apartotel La Torre & 3,744 & 7,547 \\
\hline Venta Conejo & 3,744 & 7,547 \\
\hline Venta El Moral & 3,744 & 7,547 \\
\hline R. El Escribano & 3,744 & 15,094 \\
\hline R. José Manuel & 3,744 & 15,094 \\
\hline R. Verdiales & 3,744 & 15,094 \\
\hline Venta El Agua & 3,744 & 7,547 \\
\hline Mesón Jamón & 3,744 & 9,434 \\
\hline Mesón Entrepucheros & 3,744 & 9,434 \\
\hline Pensión Bellavista & 3,744 & 7,547 \\
\hline
\end{tabular}

Tabla 2. Grado de centralidad normalizado de los actores turísticos de antequera

Fuente: elaboración propia 
La primera característica de la estructura de la red de actores turísticos de la ciudad de Antequera que se puede comprobar con los datos anteriormente expuestos, es que existe un número importante de actores que tiene un grado normalizado de centralidad de salida y de entrada muy grande, que está en torno al 100\%, además de otros dos actores que superan de forma evidente el $50 \%$ de centralidad de salida. Estos actores esta constituidos por el Ayuntamiento de la Ciudad, El Centro de Iniciativas Turisticas, la Diputacion Provincial y el Grupo de Desarrollo Rural de la comarca de Antequera y el grupo Antequera Golf. Este aspecto, junto con el gráfico de la red, pone de manifiesto que ésta se caracteriza por lo que se puede denominar un multicentrismo limitado. Es decir, un conjunto relativamente importante de actores turísticos que impulsan actuaciones turísticas conjuntas en la ciudad. Además, de forma casi mimética ocurre con el grado de centralidad de entrada, salvo para el caso significativo de la Diputación Provincial, de forma que ocupan un papel destacado en dicho indicador y son influyentes, en el sentido que los otros actores turísticos de la ciudad los demandan para realizar actividades en colaboración con ellos.

Otro aspecto destacado de la estructura de la red, es que existe un número de actores también bastante numerosos que presentan un grado de centralidad de salida y de entrada importante que se sitúa entre el $40 \%$ y el $50 \%$, dicho grupo esta formado por los hoteles del casco urbano y las agencias de viajes de la ciudad que actuan como receptivo turistico. Esta circunstancia se puede apreciar en la parte izquierda de la representación gráfica en el que se ve claramente un número de nodos con una maraña de vínculos entre ellos muy significativa, que le hace perder, hasta en cierta manera, la forma de tipo estrella a la red de actores.

Del mismo modo, en la parte más superior de la parte izquierda de la red, y con un grado de centralidad de salida y entrada que se sitúa aproximadamente entre el $30 \%$ y el $15 \%$ se encuentran otro número significativo de actores, formado por los restarantes y los hoteles y pequeñas pensiones, que aunque de manera menos acusada, presentan cierto impulso de actuaciones conjuntas para la realización de acciones turísticas en la ciudad

Y como último aspecto definitorio de las características estructurales de la red existe un número muy amplio de actores que presentan un grado normalizado de centralidad de salida y entraba muy bajo, lo que pone de manifiesto que participan poco en las interacciones que sustentan actuaciones turísticas colaborativas en la ciudad de Antequera, formado por las pensiones y ventas de restauracion que estan fuerra del casco urbano. 
Desde esta perspectiva, se puede destacar como conclusión que la red de actores turísticos de la ciudad de Antequera presenta una clara estructura de multicentrismo relativo, con cierta extensión del número de actores que impulsan a través de las relaciones con otros actores actuaciones turísticas en la ciudad.

\section{La red de actores turísticos de la ciudad de Úbeda}

El primero de los indicadores utilizados para describir las características estructurales de la red, la densidad de la red, tiene un valor del 40,80\%. Es decir, que de todas las relaciones que potencialmente podrían producirse entre los actores del Sistema Turístico de Úbeda se dan realmente casi el $41 \%$ de las mismas. Este es un indicador numéricamente importantísimo, ya que muestra que de forma real y efectiva se llevan a cabo una multitud de interacciones entre los actores turísticos de Úbeda para impulsar y desarrollar actuaciones turísticas de forma conjunta. Este indicador, sin duda, representa que existe un número alto de actores que mantienen bastantes relaciones entre ellos, como se puede comprobar de forma clara en el gráfico, en el que perfectamente se ven el número considerable de nodos que representan a los actores, muy unidos unos con otros por líneas que a su vez representan las relaciones que se producen entre ellos.

Este aspecto, a saber, el volumen importantísimo de relaciones que mantienen entre sí los actores turísticos de la ciudad de úbeda para el desarrollo de actividades turísticas conjuntas se puede ver de una manera muy nítida en la representación gráfica de dicha red que aparece en la página siguiente. 


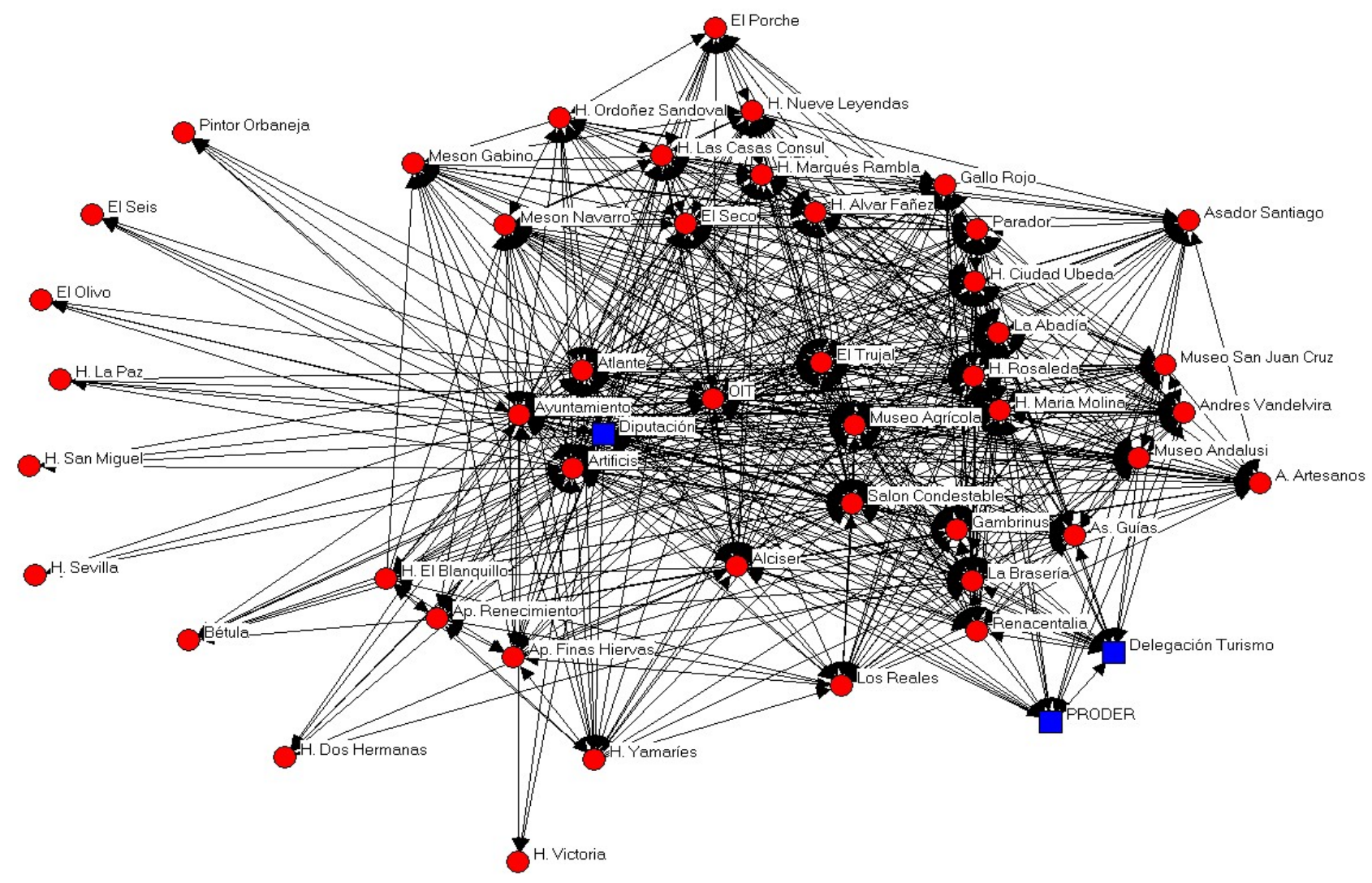

Gráfico 3. Red de actores turísticos de la ciudad de úbeda

La tabla que aparece a continuación contiene el indicador del grado normalizado de centralidad de salida y entrada de los actores de la red de relaciones de la ciudad de Úbeda

\begin{tabular}{|c|c|c|}
\hline Actor & $\begin{array}{c}\text { Grado de centralidad de } \\
\text { salida normalizado }\end{array}$ & $\begin{array}{c}\text { Grado de centralidad de } \\
\text { entrada normalizado }\end{array}$ \\
\hline Artíficis & 100 & 87,500 \\
\hline Diputación Provincial & 100 & 31,250 \\
\hline Ayuntamiento & 97,917 & 77,083 \\
\hline Atlante & 89,583 & 77,083 \\
\hline Oficina de Información & 87,500 & 37,500 \\
\hline H. Rosaleda & 68,750 & 62,500 \\
\hline Museo Agrícola & 64,583 & 72,917 \\
\hline H. María Molina & 62,500 & 60,417 \\
\hline Alciser & 58,333 & 52,083 \\
\hline Salón Condestable & 58,333 & 66,667 \\
\hline La Abadía & 65,250 & 68,750 \\
\hline Asociación de Guías & 54,167 & 52,083 \\
\hline Mesón Navarro & 52,083 & 58,333 \\
\hline
\end{tabular}




\begin{tabular}{|c|c|c|}
\hline Museo Andalusi & 52,083 & 47,917 \\
\hline R. Gambrinus & 52,083 & 60,417 \\
\hline La Brasería & 52,083 & 58,333 \\
\hline Museo San Juan de la Cruz & 52,083 & 45,833 \\
\hline H. Alvar Fáñez & 52,083 & 41,667 \\
\hline M. Andrés Vandelvira & 50,000 & 45,833 \\
\hline H. Ciudad de Úbeda & 47,917 & 52,083 \\
\hline Renacentalia & 47,917 & 43,750 \\
\hline Parador Nacional & 45,833 & 50,00 \\
\hline H. Marqués de la Rambla & 43,750 & 37,500 \\
\hline R. El Trujal & 41,667 & 66,667 \\
\hline H. Las Casas del Cónsul & 41,667 & 35,417 \\
\hline H. Nueve Leyendas & 37,500 & 39,583 \\
\hline R. Asador Santiago & 37,500 & 47,917 \\
\hline R. Los Reales & 37,500 & 45,833 \\
\hline R. El Seco & 35,417 & 58,333 \\
\hline H. Yamaríes & 35,417 & 16,667 \\
\hline Apartamentos Finas Hiervas & 35,417 & 18,750 \\
\hline Delegación de Turismo & 35,417 & 31,250 \\
\hline R. Gallo Rojo & 33,333 & 52,083 \\
\hline H. Ordóñez Sandoval & 33,333 & 29,167 \\
\hline Asociación de Artesanos & 31,250 & 41,667 \\
\hline H. El Blanquillo & 29,167 & 22,917 \\
\hline Apartamentos renacimiento & 25,000 & 27,083 \\
\hline Grupo de Desarrollo Rural & 22,917 & 25,000 \\
\hline R. El Porche & 22,917 & 29,167 \\
\hline H. Dos Hermanas & 10,417 & 12,500 \\
\hline Mesón Gabino & 6,250 & 41,667 \\
\hline R. El Seis & 2,083 & 8,333 \\
\hline H. San Miguel & 2,083 & 8,333 \\
\hline H. Sevilla & 2,083 & 6,250 \\
\hline R. Pintor Orbaneja & 0 & 10,417 \\
\hline H. La Paz & 0 & 10.417 \\
\hline H. Victoria & 0 & 6,250 \\
\hline R. El Olivo & 0 & 8,333 \\
\hline R. Bétula & 0 & 16,667 \\
\hline
\end{tabular}

Tabla 3. Grado de centralidad normalizado de los actores turísticos de úbeda Fuente: elaboración propia 
La primera característica significativa que se puede encontrar en los datos anteriores es que existe un número bastante amplio de actores que presentan un grado de centralidad de salida muy elevado, ya que como se puede comprobar 19 de los 49 actores de la ciudad superan más del 50\%. Esta situación refleja que muchos de esos actores impulsan relaciones para el desarrollo de actividades turísticas en la ciudad de Úbeda, y por tanto, se puede mantener que la estructura de la red se caracteriza, en cuanto a su centralidad, por lo que se puede denominar un policentrismo consolidado. Sobre todo, es significativo la situación de aquellos actores que superan el $70 \%$. Y algo muy parecido ocurre con el grado de centralidad de entrada, ya que prácticamente estos mismos actores presentan un grado que supera el 50\%. Este primer grupo de actores esta formado por el Ayuntamiento, la Diputacion Provincial de Jaen y las empresas de organización de visitas Atlante y Artificis.

Como se puede comprobar en la tabla anterior y en la parte derecha del gráfico, desde arriba hacia abajo, existe otro grupo de actores también, significativamente importante en cuanto al grado de centralidad que presentan. Así, este grupo de actores formado por los hoteles y restaurantes del centro historico, la Delegacion Provincial de Turismo de la Consejeria de Turismo Comercio y Deporte de la Junta de Andalucia y la Asociacion de Artesanos presentan un grado de centralidad de salida de entre el $50 \%$ y el $30 \%$, e incluso unos datos un poco más elevados en el grado de centralidad normalizado de salida.

Se puede destacar de forma clara que existe un número grande de actores que ocupan posiciones de cierta centralidad en esta red de relaciones, y que por tanto están inmersos de forma directa en el desarrollo y realización de actividades turísticas de forma conjunta en la ciudad de Úbeda.

De esta manera, y también resulta significativo, el número de actores con grado de centralidad de entrada y salida bajo es muy poco numeroso, a diferencia de lo que ocurría en las redes de las otras dos ciudades, y que esta formado por los hostales y algunos restaurantes de la periferia del casco historico.

Lo que pone de manifiesto los indicadores utilizados en el Análisis de Redes Sociales, es que la red de actores turísticos de la ciudad de Úbeda presenta una estructura de claro policentrismo, es decir, una estructura en la que está presente un número elevado de actores turísticos que impulsan vínculos con otros actores para realizar actuaciones turísticas de forma conjunta. 


\section{Conclusión}

Como fue expuesto al principio, el objetivo fundamental del trabajo consiste en determinar si existe una vinculación entre las características estructurales de las redes de los actores turísticos de las ciudades analizadas y el nivel de desarrollo turístico de las mismas. Y sobre la base de los resultados, expuestos en el apartado precedente, se puede decir que dicho objetivo se cumple, ya que se puede mantener que dicha vinculación existe. Y para ello, puede ser ilustrativo el siguiente cuadro, que recoge de forma resumida las características estructurales de las redes y el nivel de desarrollo turístico de los tres casos estudiados.

\begin{tabular}{|l|c|c|c|}
\hline \multicolumn{1}{|c|}{ Indicador } & \multicolumn{1}{|c|}{ Écija } & Antequera & Úbeda \\
\hline Densidad & $18,85 \%$ & $29,25 \%$ & $40,90 \%$ \\
\hline Estructura de Centralidad & $\begin{array}{l}\text { Único actor central (tipo } \\
\text { estrella) }\end{array}$ & Multicentrica relativa & Policéntrica consolidada \\
\hline $\begin{array}{l}\text { Índice de desarrollo } \\
\text { turístico }\end{array}$ & 10 & 38 & 47 \\
\hline
\end{tabular}

Cuadro 2. Características estructurales de las redes y nivel de desarrollo turístico de las ciudades Fuente: elaboración propia. Anuario económico de españa. 2007 (la caixa, 2007).

El cuadro anterior pone de manifiesto que la vinculación entre las características estructurales de las redes de actores turísticos y el nivel de desarrollo turístico que existe. De esta forma, se puede mantener que a medida que la red presenta una mayor densidad relacional y un número más elevado de actores con un alto grado de centralidad, el nivel de desarrollo turístico es, también, más importante. De esta forma, para los casos de estudio se demuestra que Écija es la ciudad que menor nivel de desarrollo turístico presenta y donde su red de actores turísticos se caracteriza por una baja densidad relacional y presenta una estructura de centralidad caracterizada por su forma de tipo estrella, es decir, un único y claro actor central que lidera casi en solitario las interacciones en materia turística en la ciudad.

Se puede comprobar, del mismo modo, para el caso de Antequera cuyo nivel de desarrollo turístico se incrementa que la densidad de la red se hace mayor y que la estructura de centralidad se hace múltiple, es decir, que existe un conjunto relativamente amplio de actores que impulsan y que están inmersos en múltiples interacciones para la realización de actuaciones turísticas en la ciudad, y por esos presenta una estructura multicéntrica dicha red. 
En el último de los casos, la ciudad de úbeda es la que tiene un mayor nivel de desarrollo turístico y en la que su red de actores turísticos dispone de una mayor densidad relacional, y su estructura se caracteriza por el policentrismo, es decir, un número muy elevado de actores que presentan indicadores de centralidad altos, y que por tanto, muestra que no existe un único o pocos actores centrales, sino que son muchos los actores centrales, y que por ello lideran, impulsan, y están inmersos en interacciones para la realización de actividades turísticas en la ciudad.

Lo que la fase actual del estudio de las relaciones entre los elementos que conforman los Sistemas Turísticos Locales de Úbeda, Antequera y Écija no ha podido mostrar es cuál es la dirección de la causalidad entre las características de la red y desarrollo turístico: se muestra que cuanto mayor es la dinámica de colaboración público-privada entre los actores turísticos de un territorio mayor es su nivel de desarrollo turístico. Esto es, que ambas situaciones están estrechamente relacionadas y que existe una notable correspondencia entre el desarrollo turístico de un territorio y su capital social, entendido como las redes de relaciones que una sociedad mantiene y pone en uso. Pero no es tan evidente, sin embargo, si es la dinámica de colaboración la que origina el desarrollo, o aquélla es una consecuencia de éste. Ese es el problema en que actualmente se está trabajando y será motivo de futuros estudios.

\section{Bibliografía}

Aas,Ch.; Ladkin, A. y Fletcher, J. (2005)."Stakeholder Collaboration and Heritage Management", Annals of Tourism Research, 32, pp 28-48.

Alburquerque, F. (2004). "Sistemas productivos locales: una mirada desde la política económica local para la generación de empleo", Programa AREA, Buenos Aires.

Aydalot, P. (1986). Milieux Innovateurs en Europa. GREMI. París.

Becattini, G. (1989). Modeli locali di svilupoo. II Mulino. Bolonia.

Becattini, G. (1991). “The Industrial District as a Craetive Milieu”, en Benko, G. y Dunford, M., Industrial Change and Regional Development. Belhaven Press. Londres.

Borgatti, F. F., Everett, M. G. y Freeman, C. (2002). Ucinet for Windows: Software for Social Networks Analysis. Harvard University. Harcard.

Bramwell, B. y Lane, B. (1999). "Collaboration and Partnerships for Sustainable Tourism".J ournal of Sustainable Tourism, vol 7: pp 179-181

Caravaca, I., González, G. y Silva, R. (2003). "Redes e innovación socioinstitucional en Sistemas Productivos Locales". Boletín de la AGE, 36: pp 103-115 
Caravaca, I., González, G., Méndez, R. y Silva, R. (2002). Innovación y Territorio. Análisis comparado de Sistemas Productivos Locales en Andalucía. Consejería de Economía y Hacienda. Junta de Andalucía. Sevilla.

Dredge, D. (2006). "Policy networks and the local organization of tourism". Tourism Management, 27: pp 269-280

Hanneman, R. A. y Riddle, M. (2005). Introduction to social network methods. Riverside, CA: University of California, Riverside (publicado en format digital en http://faculty.ucr.edu/ hanneman/)

Hsin-Yu Shih (2006). "Network characteristics of drive tourism destinations: An application of network analysis in tourism". Tourism Management, 27: pp 10291039.

La Caixa (2007). Anuario Económico de España. 2007. Barcelona: Servicio de Estudios de La Caixa.

Lazzaretti, M y Petrillo, E (2006). Tourism Local Systems and Networking. Elsevier, Oxford

Maillat, D. (1995). “Les milieux innovateurs". Sciences Humaines. 8: pp 15-29

Medeiros, L. y Bramwell, B. (2002). "Partnership and regional tourism in Brazil". Annals of Tourism Research. 4 vol 24: pp 1138-1164.

Michael, E (2007). Micro-Clusters and Networks: The Growth of Tourism. Elsevier, Oxford

Molina, J. L. (2001). El análisis de redes sociales. Una introducción. Ediciones bellaterra. Barcelona.

Novelli, M., Schmitz, B. y Spencer, T. (2006). "Networks, cluster and innovation in tourism: A UK experience". Tourism Management. 27: pp 1141-1152.

OMT-WTO (2001).: Cooperación entre sectores público y privado, CEOMT, Madrid.

OMT-WTO (2002).: Guía para Administraciones Locales: Desarrollo Turístico Sostenible, CEOMT, Madrid.

Pavlovich. K. (2003). "The evolution and transformation of a tourism destination networks: the Waitomo Caves, New Zealand". Tourism Management. 24: pp 203216.

Pforr, Ch. (2006). "Tourism Policy in the Making. An Australian Network Study". Annals of Tourism Research. vol 33 (1). pp 87-108.

Pulido, J. I. (2005). Criterios para una política turística sostenible en los parques naturales de Andalucía. Consejería de Turismo, Comercio y Deporte de la Junta de Andalucía. Sevilla.

Quiroga, A. (2003). Introducción al análisis de datos reticulares. Universidad Pompeu Fabra. Barcelona.

Rodríguez, J. A. y Mérida, F. (2005). Guía práctica de redes sociales. Universidad de Barcelona. Barcelona. 
Sautter, E. T. y Leisen, B. (1999). “La gestión de las partes interesadas. Un modelo de planificación turística". Annals of Tourism Research. 1: pp 109-119.

Selin, S. y Chavez, D. (1995). “Developing an Evolutionary Tourism Partnerships Model". Annals of Tourism Research. 22 (4). pp 844-856.

Vázquez, A. (1999). Desarrollo, redes e innovación. Lecciones sobre desarrollo endógeno. Pirámide. Madrid.

Vázquez, A. (2000). “Desarrollo Local y Territorio", en Pérez, B. y Carrillo, E., Desarrollo Local: Manual de Uso. ESIC. Madrid.

Vernon, N., Essex, S., Pinder, D. y Curry, K. (2005). “Collaborative Policimaking. Local Sustainable Projects". Annals of Tourism Research. vol 32 (2). pp 325-345.

Yuksel, F., Barmwell, B. y Yuksel, A. (1999). "Stakeholder interviews and tourism planning at Pamukkale, Turkey". Tourism Management. 20: pp 351-360. 